\title{
Acute hospital care in inner London: splitting from mental health services in the community
}

Acute hospital care in psychiatry has been described as inefficient and disorganised (Muijen, 1999). Worrying as it may be, this is neither new nor surprising. Following the closure of large mental institutions and the advent of community care, hospital services were supposed to provide acute in-patient care as part of a wider system. Long-term needs of patients in the community should henceforth be met by community services that would be fully equipped and resourced to undertake this task. However, it was not long before acute wards were overwhelmed by occupancy rates of $100 \%$ and above, particularly in inner cites (Powell et al, 1995). The reason for the 'bed crisis' that followed seems essentially twofold: community services were neither equipped nor resourced as required, and the number of acute beds was not adjusted to the ensuing demand. As hospital care has come to represent the only option for many patients whose needs could not be met in the community, acute wards have become overcrowded and 'a bizarre and illogical mixture ... of old and young, male and female, psychotic and depressed, retarded and agitated and voluntary and detained' (Muijen, 1999).

Although the case for provision of adequate resources is pressing, hospital services have to find alternatives to overcome, and hopefully improve, the grim situation that they currently face. Crises may not always be welcome, but at least they can bring innovation. Psychiatric services at Guy's Hospital provide mental health care to north Southwark, a deprived inner city area in London. In an attempt to address the unremitting pressure upon the local acute in-patient unit, we have implemented a system that could be of interest to other inner city mental health services, namely a clear divide between hospital and community care, with the adoption of a dedicated hospital-based, consultant-led, multidisciplinary team.

\section{The bed crisis at Guy's: separating hospital from community care}

The adult population of the catchment area (Jarman Index score of +16.25 ) is approximately 85000 , in addition to a sizeable itinerant population. Ethnic minority groups make up $25 \%$ of the population. The acute 23 bed unit opened in 1997 and resulted from the merger of two smaller but overpopulated wards, each one previously led by a different consultant and attached to different community teams. The staff of the two wards were rearranged as a single multi-disciplinary team, including an associate specialist and two senior house officers, now led by a single hospital-based consultant. The ward was allocated three beds in a specialist unit elsewhere for patients in need of intensive care. Bed occupancy exceeded $200 \%$ for several months, during which period patient turnover could rise up to 20 admissions and discharges a week. For 2 years, the unit managed about 500 admissions a year, with $20-30 \%$ of the patients occupying a bed for longer than 3 months. This was only made possible by 'outlying' patients on other units with spare capacity within the hospital, such as rehabilitation and psychogeriatric wards, while retaining the responsibility for their clinical care. An internal audit concluded that the local 'bed crisis' reflected few alternatives to hospital care as well as the use of restrictive criteria by other agencies to accept referrals. The increased number of homeless people, coupled with insufficient placements for the mentally ill in the community, contributed to both inappropriate admissions to hospital and delays in discharging patients elsewhere. Subsequent changes in mental health policy, such as the introduction of the Care Programme Approach (CPA) and Court Diversion Schemes, aggravated the crisis further.

Despite the many changes in the provision of mental health care that have taken place since the 1980s, local services had remained virtually unchanged for decades. Our approach to the problem has been to divide adult mental health services into three consultant-led teams. In addition to the hospital team, community mental health services were organised in two multi-disciplinary teams, each one dedicated to a specific part of the catchment area and led by its own community-based consultant. Communication between hospital and community teams has focused on the continuity of care for patients admitted to hospital, so that patients could be safely discharged back to their community team after provisions for their after-care had been agreed. Liaison between the 
teams has evolved into a system where sharing information, routine meetings and a clear attribution of responsibilities allow patients to have a coordinated transition

opinion \& debate

from hospital to community care, and vice-versa. Otherwise hospital and community services function independently, thereby acknowledging in their practices that they fulfil different functions, have different priorities and operate on different timescales.

\section{Principles of acute hospital care at Guy's}

The ward operates a system that is based on six main principles.

\section{Separation between hospital and community care with an effective interface}

Community and hospital teams are separate, but share common goals for patients under their care. Patients who are admitted are reviewed jointly with the referring team in clinical meetings. Moreover, the involvement of the community team continues for the duration of the patient's stay on the ward. Practical matters, service policies and coordination of services are discussed in monthly meetings with service managers and representatives of each team.

\section{Good documentation: all in writing}

All staff receive the ward handbook that explains the functional structure, standard clinical procedures and timetable of the service. An admission form ensures that essential details of the patient's clinical history, mental state and major risk factors are available from the day of admission. A list is circulated to the team every week with updated information on all patients on the ward, including status under the Mental Health Act 1983, a summary of the management plan and current medication. Decisions and any issue of significance to the management of each case are all documented scrupulously in the medical and nursing notes. A policy of 'putting things in writing' determines that communication between the hospital and community teams must be clear and easily accessible. This includes the preparation of an admission summary within a week from the date of admission, copies of which are sent to all agencies involved and to the patient's general practitioner.

\section{Full-time medical cover}

The medical team comprises one consultant psychiatrist and one associate specialist, both approved for purposes of Section 12 (2) of the Mental Health Act 1983, and two full-time senior house officers. The clinical workload is divided into two operational sub-teams. Joint teaching and supervision, on a structured and regular basis, ensures that clinical management of patients is consistent across the team. A weekly timetable was designed to offer constant medical cover and supervision, including Section 12 (2) cover, throughout the week.

\section{Goal-directed admissions}

Discharge planning starts from the day of admission. Each newly-admitted patient receives a comprehensive assessment by a senior psychiatrist the day after admission, whereby the goals of the admission and the treatment plan are determined at an early stage. Liaison with the patient's community team from the day of admission - usually directly with the patient's care coordinator - makes it possible to negotiate dates for review meetings (e.g. CPA meetings) and the likely date of discharge, to which all involved endeavour to adhere.

\section{Pre-planned clinical meetings}

The ward holds five clinical meetings a week. These include one objectives meeting and one ward round for each sub-team, in addition to one joint troubleshooting meeting on Fridays to prepare for weekends. The objectives meetings cover day-to-day matters, such as implementing treatment plans, monitoring patients' progress and setting the agenda for the ward rounds. Ward rounds, which are pre-planned and follow a well-defined time schedule, concentrate on major clinical reviews and pre-discharge meetings, including Section 117 and CPA meetings.

\section{Emphasis on staff}

Standards of care can be set at the highest level, but little will be achieved unless the team feels motivated: high morale is paramount. By promoting in-house training and teaching programmes, as well as social activities involving all staff, the unit strives to create a good work atmosphere and a strong team spirit. Members of staff find support for initiatives aiming at their professional and educational development, including seminars on clinical psychopharmacology to the nursing team. Local management has supported the organisation of 'away days', where the functioning of the service is discussed and reviewed by the entire multi-disciplinary team outside the workplace. With full 24-hour cover of the ward by agency nurses, night staff can also attend. Suggestions for innovations that meet consensus are introduced into the routine of the unit. The ward has attained a good record of recruiting and retaining staff, despite the shortage of nurses in the UK.

\section{Discovering the benefits of acute hospital care as a specialist service}

Dedicated hospital work has given the team the opportunity to perfect a set of skills and working practices aimed at the optimal use of available resources. This, in turn, has fostered a sense of team identity and boosted morale. Not only has the unit successfully managed exceedingly high rates of bed occupancy and patient 
turnover, but there are also indications that it has been providing a good service. Compared with other local acute units, where hospital and community care had not been divided, it achieved the lowest rates of untoward serious incidents and of staff sickness, as well as the shortest duration of patients' average stay in hospital. Following further reconfiguration of local services, there will be scope to compare other indices, such as clients' satisfaction and readmission rates, with those of other acute wards working under the sectorised model. However, the model has already proved sound enough to give rise to some further developments. These include the establishment of a 10-bed unit in the community, for patients who no longer require acute care but still need a therapeutic 'bridge' to independent living, followed by the implementation of a home treatment team, none of which would be likely to succeed without the support of a well-functioning acute unit. Moreover, in an effort to further improve the standards of hospital care in the area, an old facility at Guy's has been recently converted into an additional 15-bed acute unit, on the basis of the same tested principles of its larger predecessor.

The split seems to offer some further advantages. Patients who are admitted receive a 'second opinion' automatically from the hospital team as a built-in part of their care. Also, the option to alternate the care of 'difficult' cases between mental health teams could represent to nurses and doctors the opportunity for an often needed, but otherwise unavailable, respite, a refreshing benefit to a profession at high risk of burn-out (Guthrie et al, 1999). Finally, by defining professional roles within a complex system, a clear distinction between hospital and community has helped to raise standards of care at both ends. As a result of this, continuity of care could actually prove more consistent under the split model than under the sectorised model. Patients themselves might prefer, and benefit from, a change from time to time of mental health professionals involved in their care, particularly where relationships might have become strained and a fresh view of their progress might be welcome.

\section{Hospital and community care in the inner city: time for a friendly divorce?}

The closure of the large mental institutions was not accompanied by a review of the role of acute hospital services. Yet hospital treatment remains the most expensive component of mental health care. Direct costs of schizophrenia alone in England amount to over $£ 2.6$ billion a year (Knapp, 1997) and hospital care has long been known to account for most of these costs (Davies \& Drummond, 1994). However, it was not until 1998 that the Royal College of Psychiatrists set up a working party to review the main requisites of a modern acute inpatient unit (Royal College of Psychiatrists, 1998). Recommendations were made on the size, staffing and structure of acute psychiatric wards, but the final report had little to suggest on how hospital and community services should operate as part of a wider system.
If perpetuation of the 'bed crisis' is to be curtailed, mental health services have to adapt to the transformation that both urban society and principles of mental health care have undergone in previous decades. In the inner cities, where high indices of social deprivation, psychiatric morbidity and demographic changes tend to prevail, the notion of 'community' has probably become more fluid than the original proponents of community care might have anticipated. The sectorised model, where a single consultant-led team provides both hospital and community care to a designated catchment area, and which assumes a stable but largely idealised community (Wing, 1990), might no longer be the most appropriate everywhere. There is in fact little evidence to support any intrinsic superiority of the sectorised model per se, the survival of which, at least in the inner cities, might owe more to tradition than to any tangible advantage it could offer to today's community.

It remains to be formally tested whether or not hospital services that are separate from community services provide better mental health care than sectorised services. At any rate, in a changing society, newer and more productive management styles in health care are likely to emerge if health organisations are treated as complex adaptive systems (Plsek \& Wilson, 2001). Plans for a modernised mental health service hold the promise of drawing on a range of specialist services to decrease the pressure on acute in-patient units (Department of Health, 2002). Nonetheless, forthcoming changes in mental health legislation mean that in-patient services must be prepared for the countervailing challenge of managing ever more patients with behavioural rather than psychiatric problems. On balance, despite the enthusiasm for further initiatives in community care, the putative benefits of which might not always be corroborated by evidence (Holloway, 2000), the need for hospital care will probably remain unabated, particularly in the inner cities. Psychiatry may not be different from medicine at large, where many substitutes for hospital care seem to increase overall demand for services, with little impact on overall admission to hospital or costs (Hensher et al, 1999). The separation between hospital and community care in Guy's catchment area has been an attempt to offer local solutions to the local problems, an indication that mental health services can be responsive to the specific community that they cater for and structure themselves accordingly.

In any branch of medicine, hospital care is an organised, multi-disciplinary and interpersonal service (Krogstad et al, 2002). To secure consistent care for patients, strategic priority should be given to staff stability and education to build teams with collective competence and a shared ethos of responsibility. Acute hospital care in psychiatry should be no different, which probably means that, at this stage, it should be regarded as a specialist service in its own right. After years of constant changes in mental health services, tacit recognition that this is indeed the case has come, surprisingly but reassuringly, from the Department of Health itself. Newly issued guidance from the Department of Health (2002) urges that England's psychiatric wards should now 
opinion

\& debate have their own dedicated lead consultant. Perhaps, in addition to gains to patients and mental health teams, an effort to optimise hospital care could still reward mental health services by proving to be a cost-effective exercise.

\section{Declaration of interest}

None.

\section{References}

DAVIES, L. M. \& DRUMMOND, M. F. (1994) Economics and schizophrenia: the real cost. British Journal of

Psychiatry, 165 (suppl. 25), 18-21.

DEPARTMENT OF HEALTH (2002) The Mental Health Policy Implementation Guide. http://www.doh.gov.uk/nsf/ mentalhealth et al (1999) Sources of stress, psychological distress and burnout in psychiatrists. Comparison of junior doctors, senior registrars and consultants. Psychiatric Bulletin, 23, 207-212

HENSHER, M., FULOP, N., COAST, J., et al (1999) Better out than in?
Alternatives to acute hospital care. $B M J, 319,1127-1130$.

HOLLOWAY, F. (2000) Mental health policy, fashion and evidence-based practice. Psychiatric Bulletin, 24, 161-162.

KNAPP, M. (1997) Costs of

schizophrenia. British Journal of Psychiatry, 171, 509-518.

KROGSTAD, U., HOFOSS, D. \& HJORTDAHL, P. (2002) Continuity of hospital care: beyond the question of personal contact. BMJ, 324, 36-38.

MUIJEN, M. (1999) Acute hospital care: ineffective, inefficient and poorly organised. Psychiatric Bulletin, 23, 257-259.

PLSEK, P. E. \& WILSON,T. (2001) Complexity, leadership, and management in healthcare organisations. BMJ, 323,746-749.

POWELL, R., HOLLANDER, D. \& TOBINASKY R. (1995) Crisis in admission beds. British Journal of Psychiatry, 167, 765-769.

ROYAL COLLEGE OF PSYCHIATRISTS

(1998) Not just bricks and mortar. Report of the Royal College of

Psychiatrists Working Party on the size, staffing, structure, siting, and security of new acute adult psychiatric in-

patient units. Council Report CR62 London: Royal College of Psychiatrists.

WING, J. K. (1990). The functions of asylum. British Journal of Psychiatry, $157,822-827$.

*Luiz Dratcu Consultant Psychiatrist, Alistair Grandison Associate Specialist in Psychiatry, Antony Adkin In-patient Services Manager, York Clinic, Guy's Hospital, South London \& Maudsley NHS Trust, London SE1 3RR 